\title{
Synaptic connections to individual tibialis anterior motoneurones in man
}

\author{
PETER ASHBY A ND DUA NE ZILM \\ From Toronto Western Hospital and Addiction Research Foundation, Toronto, Canada
}

SU M M AR Y The characteristics of post-synaptic potentials in a single human motoneurone can be derived from the profiles of post-stimulus time histograms (PSTH) of that neurone when it is firing rhythmically. We have used this method to explore the synaptic connections to individual tibialis anterior motoneurones in man. Agonist group 1 volleys produced short latency excitation of $85 \%$ of tibialis anterior motoneurones probably representing the 1a EPSP. Excitation, at a latency of $70 \mathrm{~ms}$, is attributed to a long loop reflex. Antagonist group 1 volleys produced short latency inhibition of $65 \%$ of tibialis anterior motoneurones probably representing the 1a IPSP. Excitation, at a latency of $97 \mathrm{~ms}$, may represent spino-bulbo-spinal reflex. None of these effects are due to the excitation of cutaneous afferent nerve fibres.

When a neurone is firing rhythmically, the effects of an afferent volley on that neurone can be determined from the way in which the volley alters the neurone's probability of firing. For volleys evoked by a recurrent external stimulus, these changes in probability can be expressed in the form of a post-stimulus time histogram (PSTH). The way in which the profile of the PSTH reflects the shape of the post-synaptic potential is complex (Bryant et al., 1973) and has been the subject of mathematical analysis (Knox, 1974; Knox and Poppele, 1977) and computer simulation (Knox et al., 1977). As a first approximation, and provided that the combined slope of the synaptic potential and the spontaneous membrane potential remains positive, the PSTH profile may be considered as the first derivative of the shape of the post-synaptic potential (Knox and Poppele, 1977). Post-synaptic potentials reconstructed from the PSTH in this way are in general accord with the findings of intracellular recordings (Knox et al., 1977).

The firing pattern of a single human motoneurone can be extracted by identifying each occurrence of its motor unit action potential in a recording obtained with an intramuscular needle electrode (Ashby and LaBelle, 1977). It is, therefore, possible to generate the PSTH of a human motoneurone after a variety of afferent volleys

Address for reprint requests: Dr Peter Ashby, 25 Leonard Avenue, Toronto, Ontario, M5T 2R2, Canada.

Accepted 16 February 1978 and, in this way, to derive some of the characteristics of the underlying post-synaptic potentials. The afferent connections to soleus (Ashby and and LaBelle, 1977) and to first dorsal interosseous motoneurones (Stephens et al., 1976) have been examined in this way.

This report deals with the afferent connections to human tibialis anterior motoneurones.

\section{Methods}

The method has been described previously (Ashby and LaBelle, 1977). The subjects lay supine. Stimuli were delivered to the peroneal nerve at the head of the fibula with a bipolar surface electrode. In order to deliver an agonist group 1 afferent volley, the stimulus current was adjusted so that it was just below the level required to produce a visible contraction of the tibialis anterior. A second bipolar electrode was used to deliver stimuli to the popliteal nerve in the popliteal fossa. The latency of the maximum $\mathrm{H}$ reflex (recorded with surface electrodes over soleus) was noted. In order to deliver an antagonist group 1 afferent volley, the stimulus current was then reduced until the $\mathbf{H}$ reflex did not occur (except during rare spontaneous fluctuations in central excitability). The effects of cutaneous stimuli were examined separately by positioning the stimulating electrode at various sites on the leg.

The action potentials of voluntarily activated tibialis anterior motor units were recorded with a 
concentric needle electrode inserted into the belly of the left tibialis anterior. The signals were amplified 1000 to 5000 times and displayed using standard electromyographic equipment (Tektronix $5 \mathrm{~A} 22 \mathrm{~N}$ amplifier with band pass $30 \mathrm{~Hz}$ to $0.1 \mathrm{MHz}$ ) and passed through an audio amplifier to a loudspeaker. The subject was provided with this audio and visual feedback.

The needle electrode was positioned close to a motor unit in tibialis anterior and the subject instructed to keep the unit discharging steadily while approximately 250 square-wave stimuli (100$500 \mu$ s duration) at $1013 \mathrm{~ms}$ intervals were delivered to one or other muscle nerve or to the skin. The EMG signal and a pulse generated by the stimulator were recorded using a Hewlett-Packard 3960 FM tape recorder. A number of units were examined in each subject by moving the needle to various sites in the muscle.

The analysis was performed from the tape. The action potentials of an individual motor unit were selected with the help of a window discriminator and a delay line. The characteristics of the motor unit action potential were obtained (by repeated averaging) and the interval data, including the PSTH, generated using a PDP12 computer. Because the window discriminator generated a pulse at the negative peak of the motor unit action potential, all latencies in the PSTH were corrected by subtracting the rise time of the motor unit action potential.

The details of this analysis, including certain important points that must be considered during the collection and interpretation of the PSTH, have been discussed previously (Ashby and LaBelle, 1977).

Student's (unpaired) $t$ test and the correlation coefficient were used in the statistical analysis.

\section{Results}

Studies were carried out on 10 normal subjects (seven male, three female, mean age $33 \mathrm{yr}$ ). In all, 37 tibialis motor units were isolated. The action potentials of these motor units had a mean amplitude of $1018 \mu \mathrm{V}$, a mean rise time (to the negative peak) of $4.2 \mathrm{~ms}$, and a mean duration of $16 \mathrm{~ms}$. The mean of the firing rates was 8.0 per second.

RESPONSE OF TIBIALIS MOTONEURONES TO GROUP 1 PERONEAL NERVE VOLLEYS

Agonist group 1 volleys resulted in a peak of increased impulse density in the PSTH of 22 $(85 \%)$ of the 26 tibialis motor units examined (Fig. 1). The onset of this change in impulse

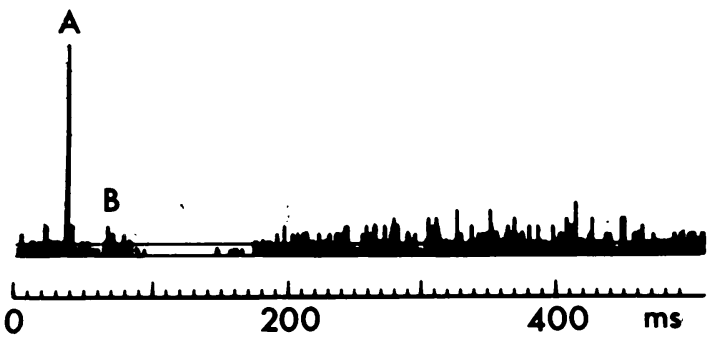

Fig. 1 Post-stimulus time histogram (PSTH) of a tibialis anterior motor unit after group 1 peroneal nerve volleys. In this and subsequent figures the horizontal line denotes the mean of the 512 post-stimulus bins. There is an early peak of increased impulse density $(A)$ with a latency of $27 \mathrm{~ms}$ and a duration of $3 \mathrm{~ms}$ above the mean level. The subsequent period of reduced impulse density is interrupted by a smaller peak at $57 \mathrm{~ms}(B)$. All the quoted latencies have been corrected for the rise time $(9 \mathrm{~ms})$ of the motor unit action potential.

density had a mean latency of $31.3 \mathrm{~ms}$. This latency was correlated with the latency (mean $29 \mathrm{~ms})$ to the onset of the soleus $\mathrm{H}$ reflex $(r=0.62$; $\mathbf{P}<0.01$ ).

The mean ratio of the height of the peak of increased impulse density to the mean height of the 512 post-stimulus bins was 9.0 (indicating that there is a nine-fold increase in the probability of a motoneurone firing at this interval after the stimulus).

The mean duration of this period of increased impulse density (measured as the number of contiguous bins in the region of the peak with contents higher than the average) was $4.7 \mathrm{~ms}$. In most instances, however, the peak was roughly "bell shaped" with the main increases in impulse density making up the peak restricted to the one or two central bins of the cluster.

This early period of increased impulse density was not observed in the PSTH of four tibialis motor units. The characteristics of the action potentials and the firing rates of these units were not significantly different from the others.

A period of reduced impulse density usually followed the initial peak. In 15 of the 26 units $(58 \%)$ a second, smaller, peak of increased impulse density could be distinguished. This peak had a mean latency of $70 \mathrm{~ms}$.

RESPONSE OF TIBIALIS ANTERIOR MOTONEURONES TO GROUP 1 POPLITEAL NERVE VOLLEYS

Antagonist group 1 volleys resulted in a period of reduced impulse density in the PSTH of $17(65 \%)$ of the 26 units examined (Fig. 2). The mean onset of this period was $33.6 \mathrm{~ms}$ and the mean termina- 


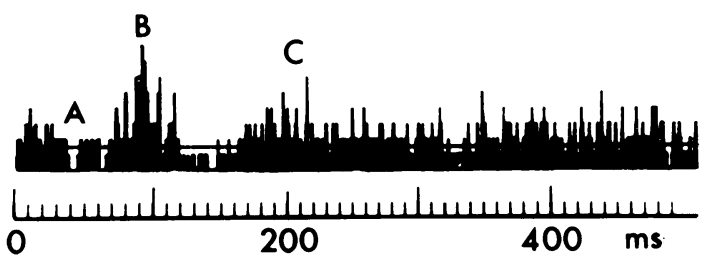

Fig. 2 PSTH of a tibialis anterior motor unit after group 1 stimulation of the popliteal nerve. There is a period of reduced impulse density from 35 to $45 \mathrm{~ms}$ (A) followed by a period of increased impulse density with a peak at $90 \mathrm{~ms}(B)$. Periodicity effects are seen at intervals corresponding to the mean interspike interval $(103 \mathrm{~ms})$ of that motor unit $(C)$. All the quoted latencies have been corrected for the rise time $(1.0 \mathrm{~ms})$ of the motor unit action potential.

tion $58.6 \mathrm{~ms}$. In 23 units $(88 \%)$ there was a peak of increased impulse density in the PSTH with a mean latency of $97 \mathrm{~ms}$. This latency was correlated $(r=0.6 ; \mathrm{P}<0.01)$ with the mean interspike interval of the unit (mean $128 \mathrm{~ms}$ ).

\section{RESPONSE OF TIBIALIS ANTERIOR MOTONEURONES} TO CUTANEOUS VOLLEYS

To determine whether the excitation of local cutaneous afferent nerve fibres under the stimulating electrode could account for any of the changes in the PSTH, stimuli of the same magnitude as those used to deliver a group 1 volley (approximately twice the detectable threshold) were delivered to the skin a few centimetres on either side of the lateral peroneal and popliteal nerves. Such stimuli had no effect on the PSTH of tibialis motoneurones.

Painful stimuli delivered to the great toe (at approximately four times the detectable threshold) caused facilitation of tibialis anterior motoneurones with mean latency of $83.5 \mathrm{~ms}$ (Fig. 3).

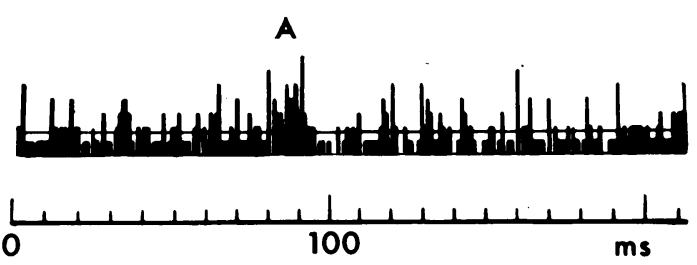

Fig. 3 PSTH of a tibialis anterior motoneurone after stimulation of the big toe at four times the level at which the stimulus was just detectable. A period of increased impulse density occurs with a peak at $83 \mathrm{~ms}(A)$. This latency has been corrected for the rise time $(4 \mathrm{~ms})$ of the motor unit action potential.

\section{Discussion}

The early peak of increased impulse density in the PSTH of tibialis anterior motoneurones after group 1 peroneal nerve volleys is of appropriate latency to represent the 1a EPSP. Alterations in impulse density in the PSTH reflect approximately the first derivative of the contour of post-synaptic potentials, provided that the combined slope of the synaptic potential and the spontaneous membrane potential remains positive (Knox, 1974; Knox and Poppele, 1977). A description of the shape of the rising phase of the EPSP can, therefore, be obtained by integrating the PSTH (Fig. 4), and the duration of the early period of increased impulse density in the PSTH represents the rise time of the human 1a EPSP.

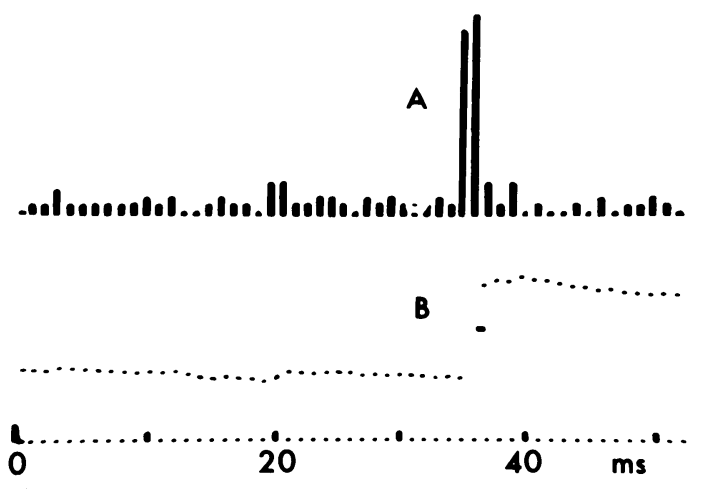

Fig. 4 Description of the rising phase of the composite la EPSP produced by an agonist group 1 volley in a single human tibialis anterior motoneurone $(B)$ obtained by integrating the initial portion of the PSTH illustrated in Fig. $1(A)$. The integral does not provide an accurate description of the falling phase of an EPSP (see text).

The mean duration of this period of increased impulse density in the PSTH of human tibialis motoneurones was $4.7 \mathrm{~ms}$. This is longer than the rise time (approximately $1.5 \mathrm{~ms}$ ) of the composite 1a EPSP recorded with intracellular electrodes in tibialis motoneurones of the cat (Eccles et al., 1957a). However, the duration of the early period of increased impulse density was measured as the number of contiguous bins in the region of peak with contents higher than the mean of the 512 post-stimulus bins, whereas the main increases in impulse density occupied the one or two central bins of this cluster. If the duration of the peak of increased impulse density had been measured as the number of contiguous bins above the variance of the mean of the 512 pre-stimulus bins, the estimate of the rise time would be considerably 
reduced. Our present computer programmes could not accomplish this, but it would be reasonable to adopt this method of measurement in future studies.

The amplitude of an EPSP is reflected in the PSTH profile both by the height of the PSTH peak and by the duration of the subsequent period of reduced impulse expressed as a proportion of the mean interspike interval (Ashby and LaBelle, 1977; Knox and Poppele, 1977). The substantial early peaks observed in this study indicate that the 1a EPSP in human tibialis anterior motoneurones is large, but the proportion of the mean interspike interval occupied by the subsequent period of reduced impulse density could not be established clearly in many instances. As an alternative method of estimating the amplitude of 1a EPSP in man, group 1 volleys were interpolated at various intervals after a spontaneously occurring motoneurone discharge. These studies (still in progress) indicate that the composite 1a EPSP is large enough to bring the membrane potential of human tibialis anterior motoneurones to threshold at least in the last half of the interspike interval. If the subthreshold excursion of the interspike membrane potential of a rhythmically firing human motoneurone is about $15 \mathrm{mV}$, as it is in cat motoneurones (Schwindt and Calvin, 1973) and, if the reduction in threshold during the interspike interval is approximately $5 \mathrm{mV}$ (Calvin, 1974, 1975), the amplitude of the composite 1a EPSP in human tibialis anterior motoneurones must be in the region of $5 \mathrm{mV}$. The amplitude of the comparable composite 1a EPSP recorded in cat tibialis motoneurones with intracellular electrodes is $4.5 \mathrm{mV}$ (Eccles et al., 1957a).

The PSTH profile can not provide a description of the falling phase of an EPSP where the combined slope of the synaptic potential and the spontaneous membrane potential is negative (Knox, 1974; Knox and Poppele, 1977). For this reason an alternative method of exploring the falling phase of the EPSP has been devised. A group 1 volley is interpolated after a spontaneous motoneurone discharge sufficiently early to ensure that the EPSP just fails to bring the membrane potential to threshold. If this volley is then preceded, at various intervals, by a conditioning group 1 volley, temporal summation will bring the membrane potential to threshold and the falling phase of the EPSP can be explored. Preliminary studies of this type suggest (if presynaptic variables can be discounted) that the composite 1a EPSP in tibialis anterior motoneurones in man has largely decayed $10 \mathrm{~ms}$ after its onset.
The small second peak (occurring at a latency of $70 \mathrm{~ms}$ ) probably represents the rising phase of a polysynaptic EPSP rather than the rising phase of an IPSP. A burst of EMG with similar latency (70-80 $\mathrm{ms})$ has been observed after dynamic stretch of tibialis anterior in man (Kearney and Chan, personal communication). Similar late responses to muscle stretch (attributed to transcortical loops) have been recorded from flexor hallucis longus with a latency of $75-90 \mathrm{~ms}$, thumb flexor muscles, with a latency of $45 \mathrm{~ms}$ (Marsden et al., 1976), and forearm muscles with a latency of 55-60 ms (Lee and Tatton, 1975). The $70 \mathrm{~ms}$ PSTH peak is, therefore, tentatively attributed to facilitation from a long loop reflex pathway.

An IPSP appears in the PSTH as a period of reduced impulse density followed by a period of increased impulse density (Bryant et al., 1973; Knox and Poppele, 1977). Group 1 volleys delivered to the antagonist muscle afferent nerves produced a period of reduced impulse density of appropriate latency to represent the 1a IPSP. This was followed by a period of increased impulse density with a peak at 97 ms. However, the combined duration of the gap and the subsequent peak is much longer than the duration of the 1a IPSP in cat motoneurones (Coombs et al., 1955; Araki et al., 1960) or the presumed duration of the 1a IPSP in man (Tanaka, 1972). Furthermore, the peak sometimes occurred without the preceding gap. The peak is, therefore, considered to be a separate facilitatory effect. This facilitation must arise from muscle afferent fibres as it was observed when group 1 stimuli were delivered to the antagonist muscle nerve, but not when they were delivered to the neighbouring skin. The latency is too long for this to represent facilitation arising from direct electrical stimulation of $1 \mathrm{~b}$ afferent fibres (Laporte and Lloyd, 1952; Eccles et al., 1957b). Shimamura et al. (1964) described facilitation of tibialis motoneurones with a latency of 70-80 ms, resulting from stimulation of low threshold afferent fibres in the popliteal nerve in man and attributed this to spino-bulbo-spinal reflex pathways. The facilitation we observed appears to be similar.

The effects of cutaneous stimuli were not explored systematically. It is clear that noxious stimuli applied to the big toe can result in a late $(84 \mathrm{~ms})$ facilitation of tibialis anterior motoneurones. This may represent a long loop reflex (Meier-Ewert et al., 1972) or a spinal flexion reflex (Hagbarth, 1960; Shahani and Young, 1971; Gassel and Ott, 1973). There appears to be no contribution to the PSTH of tibialis motoneurones from the activation of cutaneous afferent 
fibres under the stimulating electrode during group 1 stimulation of the peroneal or popliteal nerves. The short latency inhibition of small hand muscle motoneurones from cutaneous afferent fibres (Caccia et al., 1973; Stephens et al., 1976) may be unique to the upper limb.

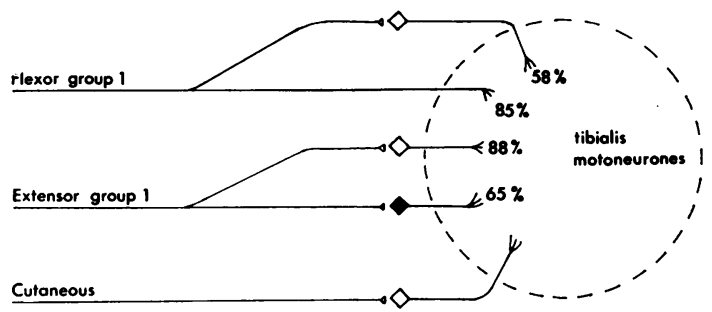

Fig. 5 Diagram of the synaptic connections to human tibialis anterior motoneurones based on the observations of this study. Dark neurones have inhibitory synapses. All other synapses are facilitatory. Agonist group 1 volleys produce short latency facilitation of $85 \%$ of tibialis anterior motoneurones, probably representing the Ia IPSP. A longer latency (70 ms) facilitation was observed in $58 \%$ of neurones possibly representing a long loop reflex. Antagonist group 1 volleys produce short latency inhibition of $65 \%$ of tibialis anterior motoneurones, probably representing the la IPSP. A spino-bulbo-spinal reflex pathway may account for the later facilitation of $88 \%$ of neurones. Noxious cutaneous stimuli are capable of producing late facilitation of tibialis motoneurones.

A diagram of the synaptic connections to human tibialis anterior motoneurones based on the observations of this study is presented in Fig. 5 . The present technique can provide data equivalent to those obtained from intracellular recordings. In fact, this technique may be a more sensitive way of detecting small post-synaptic potentials (Bryant et al., 1973; Knox and Poppele, 1977). Post-synaptic potentials produced by single afferent nerve fibres could be examined by generating a PSTH from spikes recorded from single afferent fibres (Mendell and Henneman, 1968; Vallbo, 1973). The size and shape of human post-synaptic potentials may be estimated in greater detail when the present method is supplemented by studies using interpolated single or double stimuli. Since many motoneurones are spontaneously active the PSTH probably provides the most realistic dynamic description of the effects of an input on a functioning neuronal network.

We thank the Connaught Foundation for financial support. The illustrations were photographed by the Audio Visual department of the Toronto
Western Hospital. Nancy Bennett prepared the manuscript.

\section{References}

Araki, T., Eccles, J. C., and Ito, M. (1960). Correlation of the inhibitory postsynaptic potential of motoneurones with the latency and time course of inhibition of monosynaptic reflexes. Journal of Physiology, 154, 354-377.

Ashby, P., and LaBelle, K. (1977). Effects of extensor and flexor group 1 afferent volleys on the excitability of individual soleus motoneurones in man. Journal of Neurology, Neurosurgery, and Psychiatry, 40, 910-919.

Bryant, H. L., Marcos, A. R., and Segundo, J. P. (1973). Correlations of neuronal spike discharges produced by monosynaptic connections and by common inputs. Journal of Neurophysiology, 36, 205-225.

Caccia, M. R., McComas, A. J., Upton, A. R. M., and Blogg, T. (1973). Cutaneous reflexes in small muscles of the hand. Journal of Neurology, Neurosurgery, and Psychiatry, 36, 960-977.

Calvin, W. H. (1974). Three modes of repetitive firing and the role of threshold time course between spikes. Brain Research, 69, 341-346.

Calvin, W. H. (1975). Generation of spike trains in CNS neurons. Brain Research, 84, 1-22.

Coombs, J. S., Eccles, J. C., and Fatt, P. (1955). The inhibitory suppression of reflex discharges from motoneurones. Journal of Physiology, 130, 396-413.

Eccles, J. C., Eccles, R. M., and Lundberg, A. (1957a). The convergence of monosynaptic excitatory afferents on to many different species of alpha? motoneurones. Journal of Physiology, 137, 22-50.

Eccles, J. C., Eccles, R. M., and Lundberg, A. (1957b). Synaptic actions on motoneurones caused by impulses in Golgi tendon organ afferents. Journal of Physiology, 138, 227-252.

Gassel, M. M., and Ott, K. H. (1973). Patterns of reflex excitability change after widespread cutaneous stimulation in man. Journal of Neurology, Neurosurgery, and Psychiatry, 36, 282-287.

Hagbarth, K. E. (1960). Spinal withdrawal reflexes in the human lower limbs. Journal of Neurology, Neurosurgery, and Psychiatry, 23, 222-227.

Knox, C. K. (1974). Cross-correlation functions for a neuronal model. Biophysical Journal, 14, 567-582.

Knox, C. K., and Poppele, R. E. (1977). Correlation analysis of stimulus evoked changes in excitability of spontaneously firing neurons. Journal of Neurophysiology, 40, 616-625.

Knox, C. K., Kubota, S., and Poppele, R. E. (1977). A determination of excitability changes in DSCT neurons from spike train analysis. Journal of Neurophysiology, 40, 626-646.

Laporte, Y., and Lloyd, D. P. C. (1952). Nature and significance of the reflex connections established by large afferent fibers of muscular origin. American Journal of Physiology, 169, 609-621. 
Lee, R. G., and Tatton, W. G. (1975). Motor responses to sudden limb displacements in primates with specific CNS lesions and in human patients with motor system disorders. Canadian Journal of Neurological Sciences, 2, 285-293.

Marsden, C. D., Merton, P. A., and Morton, H. B. (1976). Stretch reflex and servo action in a variety of human muscles. Journal of Physiology, 259, 531560.

Meier-Ewert, K., Hümme, U., and Dahm, J. (1972). New evidence favouring long loop reflexes in man. Archiv für Psychiatrie und Nervenkrankheiten, 215, 121-128.

Mendell, L. M., and Henneman, E. (1968). Terminals of single la fibers: distribution within a pool of 300 homonymous motor neurons. Science, 160, 96-98.

Schwindt, P. C., and Calvin, W. H. (1973). Equivalence of synaptic and injected current in determining the membrane potential trajectory during motoneuron rhythmic firing. Brain Research, 59, 389394.
Shahani, B. T., and Young, R. R. (1971). Human flexor reflexes. Journal of Neurology, Neurosurgery, and Psychiatry, 34, 616-627.

Shimamura, M., Mori, S., Matsushima, S., and Fujimori, B. (1964). On the spino-bulbo-spinal reflex in dogs, monkeys and man. Japanese Journal of Physiology, 14, 411-421.

Stephens, J. A., Usherwood, T. P., and Garnett, R. (1976). Technique for studying synaptic connections of single motoneurones in man. Nature, 263, 343344.

Tanaka, R. (1972). Activation of reciprocal la inhibitory pathway during voluntary motor performance in man. Brain Research, 43, 649-652.

Vallbo, A. B. (1973). Muscle spindle afferent discharge from resting and contracting muscles in normal human subjects. In New Developments in Electromyography and Clinical Neurophysiology. Vol. 3, pp. 251-262. Edited by J. E. Desmedt. Karger: Basel. 